\title{
THE TEMPORAL EVOLUTION OF A SYSTEM IN COMBUSTION THEORY
}

\author{
K. K. TAM ${ }^{1}$
}

(Received 12 November 1981; revised 19 February 1982)

\begin{abstract}
A model governing the combustion of a material is considered. The model consists of two non-linear coupled parabolic equations with initial and boundary conditions. An approximation for the rate of reactant consumption is made to enable the system to be treated by Laplace transform. Three simple geometries are considered; namely, an infinite slab, an infinite circular cylinder and a sphere. The results obtained are then compared with numerical solutions for some specific values of the parameters. There is good agreement over the time duration for which numerical work was performed.
\end{abstract}

\section{Introduction}

A simple model governing the combustion of a material can be formulated in non-dimensional form as follows,

$$
\begin{aligned}
& \frac{\partial \theta}{\partial t}=\nabla^{2} \theta+H \chi \exp \left(\frac{\alpha \theta}{\alpha+\theta}\right), \\
& \frac{\partial \chi}{\partial t}=\nabla^{2} \chi-\varepsilon \chi \exp \left(\frac{\alpha \theta}{\alpha+\theta}\right), \\
& \theta(x, 0)=h(x), \quad \theta=0 \text { on } \partial D,
\end{aligned}
$$

\footnotetext{
'Department of Mathematics, McGill University, 805 Sherbrooke Street West, Montreal, Quebec H3A 2K6, Canada.

C. Copyright Australian Mathematical Society 1983
} 


$$
\chi(x, 0)=g(x), \quad \frac{\partial \chi}{\partial \nu}=0 \text { on } \partial D
$$

Here $\theta$ is the temperature, $\chi$ the concentration of the combustible material; $x$ and $t$ are respectively the spatial and time variables. These variables are supposed to have been nondimensionalized. The parameters $H$ and $\alpha$ are positive and $\varepsilon=$ $\exp (-\alpha)$. Typically, the value of $\alpha$ is between 10 and 100 so that $\varepsilon \ll 1$. The equations ( 1 ) and ( 2 ) are considered in a bounded domain $D$ with initial and boundary values given in (3) and (4). The derivation of the above system can be found in Frank-Kamenetskii [1], and discussions on the system can be found in Gelfand [2], Parks [4], and Sattinger [5], among others. It is known that if the initial concentration and the initial temperature are small, $\chi$ decays very slowly and $\theta$ remains of order one. We shall refer to such a situation as subcritical. However, if the initial temperature and/or the initial concentration is sufficiently large, $\chi$ decays rapidly and $\theta$ becomes extremely large before both finally decay to zero. We shall refer to such a situation as supercritical. In using the terms subcritical or supercritical, we do not imply that we have defined the critical conditions when reactant consumption is taken into account. However, these terms do describe clearly and effectively the two distinctly different situations. For the subcritical case, Sattinger obtained an asymptotic development for $\theta$ and $\chi$ based on $\varepsilon \ll 1$.

Recently, Tam [6] used a comparison theorem to construct upper and lower solutions; and in [7], [8] used an integral equations approach to consider the influence of the initial data. An attempt was also made in [7] to construct an approximate description of the temporal evolution of $\theta$ in the super-critical case. In [3] Kassoy used the method of matched asymptotic expansions to study the rapid transient phenomena in a similar system but only the spatially homogeneous problem was considered.

In this note, we wish to examine the extremely rapid transient behaviour of the system in the supercritical case for an infinite slab, an infinite circular cylinder and a sphere, for the case of zero initial temperature. Because of the nonlinearity, analytical treatment of the system is difficult. However, the problem has been studied for some time and it is known that $\chi$ is almost independent of the spatial variables (see [3]). We make use of this observation to construct approximate descriptions for $\theta$ and $\chi$ as follows. We first combine (1) and (2) to form a linear equation which is solved by using the Laplace transform. Then, assuming an initial approximation for $\chi$ which is spatially homogeneous, we solve for $\theta$, from which an improved approximation for $\chi$ is obtained. The only assumption used is that the solution is supercritical. The approximation obtained is not limited to small $t$. Indeed, the multiple time scale effect is apparent in the improved approximation for $\chi$. 


\section{Approximation for $\theta$ and $\chi$ for the slab}

We take $\nabla^{2}=\partial^{2} / \partial x^{2}, 0<x<1, h(x)=0$ and $g(x)=N$, a constant. If we write $\varepsilon \theta+H \chi=\varphi$, equations (1) and (2) combine to give

$$
\frac{\partial \varphi}{\partial t}=\frac{\partial^{2} \varphi}{\partial x^{2}}
$$

subject to

$$
\varphi(x, 0)=H N
$$

and

$$
\varphi(0, t)=\varphi(1, t)=H_{\chi_{s}}(t),
$$

where $\chi_{s}(t)$ is the surface concentration to be determined. Using a Laplace transform with respect to $t$, and using a bar to denote the transformed quantities, we readily obtain

$$
\bar{\varphi}(p, x)=\frac{H N}{p}\left(\frac{\cos i \sqrt{p} / 2-\cos i \sqrt{p}\left(x-\frac{1}{2}\right)}{\cos i \sqrt{p} / 2}\right)+\frac{H \bar{\chi}_{s} \cos i \sqrt{p}\left(x-\frac{1}{2}\right)}{\cos i \sqrt{p} / 2},
$$

where

$$
\mathcal{L}(\varphi)=\bar{\varphi}=\int_{0}^{\infty} \varphi(x, t) e^{-p t} d t
$$

and

$$
\mathfrak{L}^{-1}(\bar{\varphi})=\varphi=\frac{1}{2 \pi i} \int_{c-i \infty}^{c+i \infty} \bar{\varphi}(p, x) e^{p t} d p
$$

We have

$$
\begin{aligned}
& \mathcal{L}^{-1}\left(\frac{\cos i \sqrt{p} / 2-\cos i \sqrt{p}\left(x-\frac{1}{2}\right)}{p \cos i \sqrt{p} / 2}\right) \\
& =\sum_{n=0}^{\infty} \frac{(-1)^{n} 4 \cos \left[(2 n+1) \pi\left(x-\frac{1}{2}\right)\right]}{(2 n+1) \pi} e^{-(2 n+1)^{2} \pi^{2} t} \\
& \equiv \varphi_{1}(x, t),
\end{aligned}
$$

and thus

$$
\varepsilon \theta(x, t)+H \chi(x, t)=H N \varphi_{1}(x, t)+\mathfrak{e}^{-1}\left(\frac{H \bar{\chi}_{s} \cos i \sqrt{p}\left(x-\frac{1}{2}\right)}{\cos i \sqrt{p} / 2}\right) .
$$


If we consider (8) as an expression defining $\theta(x, t)$ in terms of $\chi$, we can re-write (2) and (4) as an integral equation involving $\chi$ only. However, the resulting nonlinear integral equation remains intractable and, to proceed further, we would have to start an iteration process. While the number of times we can iterate in practice is extremely limited, we do have the knowledge that the iteration is convergent since the function $\exp (\alpha \theta / \alpha+\theta)$ is uniformly bounded. In view of the above, we shall start with an initial approximation for $\chi$, and use (8) to obtain an approximation for $\theta$. An improved description for $\chi$ can then be obtained from (2).

We observe that as a first approximation, the concentration $\chi$ can be considered as spatially homogeneous. Thus, we assume $\chi(x, t) \sim \chi_{s}(t)$. To see what form $\chi_{s}(t)$ should take, we obtain from (2) the expression

$$
\chi_{s}(t) \simeq N \exp \left\{-\varepsilon \int_{0}^{t} \exp \left(\frac{\alpha \theta\left(\frac{1}{2}, \tau\right)}{\alpha+\theta\left(\frac{1}{2}, \tau\right)}\right) d \tau\right\},
$$

where we have avoided inconsistency regarding the spatial independence of $\chi$ by taking $x=\frac{1}{2}$. Clearly, if $\theta\left(\frac{1}{2}, \tau\right) \gg \alpha, \chi_{s}(t)$ decays as $\exp (-t)$, whereas if $\theta\left(\frac{1}{2}, \tau\right)$ is not exponentially large, $\chi_{s}(t)$ decays as $\exp (-\varepsilon t)$. Since we are interested in the super-critical case, we suppose $H N$ is sufficiently large for the former case to happen. We shall therefore take as our first approximation for $\chi$ as

$$
\chi_{s}=N e^{-t}
$$

This approximation is a good one as long as $\theta\left(\frac{1}{2}, \tau\right) \gg \alpha$. From our previous studies, [9], we have seen that $\theta\left(\frac{1}{2}, \tau\right)$ remains large until $H \chi \sim \delta_{e}(\alpha)$, which is about $2 \times 10^{-5}$ for $\alpha=20$, for all three simple geometries. When that stage is reached, $\chi$ is so small that whether its further decay is as $\exp (-t)$ or $\exp (-\varepsilon t)$ becomes unimportant as far as the explosive transient phenomenon is concerned. Thus, we have

$$
\bar{\chi}_{s}(p)=\frac{N}{p+1}
$$

and hence

$$
\begin{aligned}
& \mathcal{L}^{-1}\left(\frac{H \bar{\chi}_{s} \cos i \sqrt{p}\left(x-\frac{1}{2}\right)}{\cos i \sqrt{p} / 2}\right)=\frac{H N \cos \left(x-\frac{1}{2}\right)}{\cos \frac{1}{2}} e^{-t} \\
& -H N \sum_{n=0}^{\infty} \frac{(-1)^{n} 4(2 n+1) \pi}{(2 n+1)^{2} \pi^{2}+1} \cos \left[(2 n+1) \pi\left(x-\frac{1}{2}\right)\right] e^{-(2 n+1)^{2} \pi^{2} t}
\end{aligned}
$$




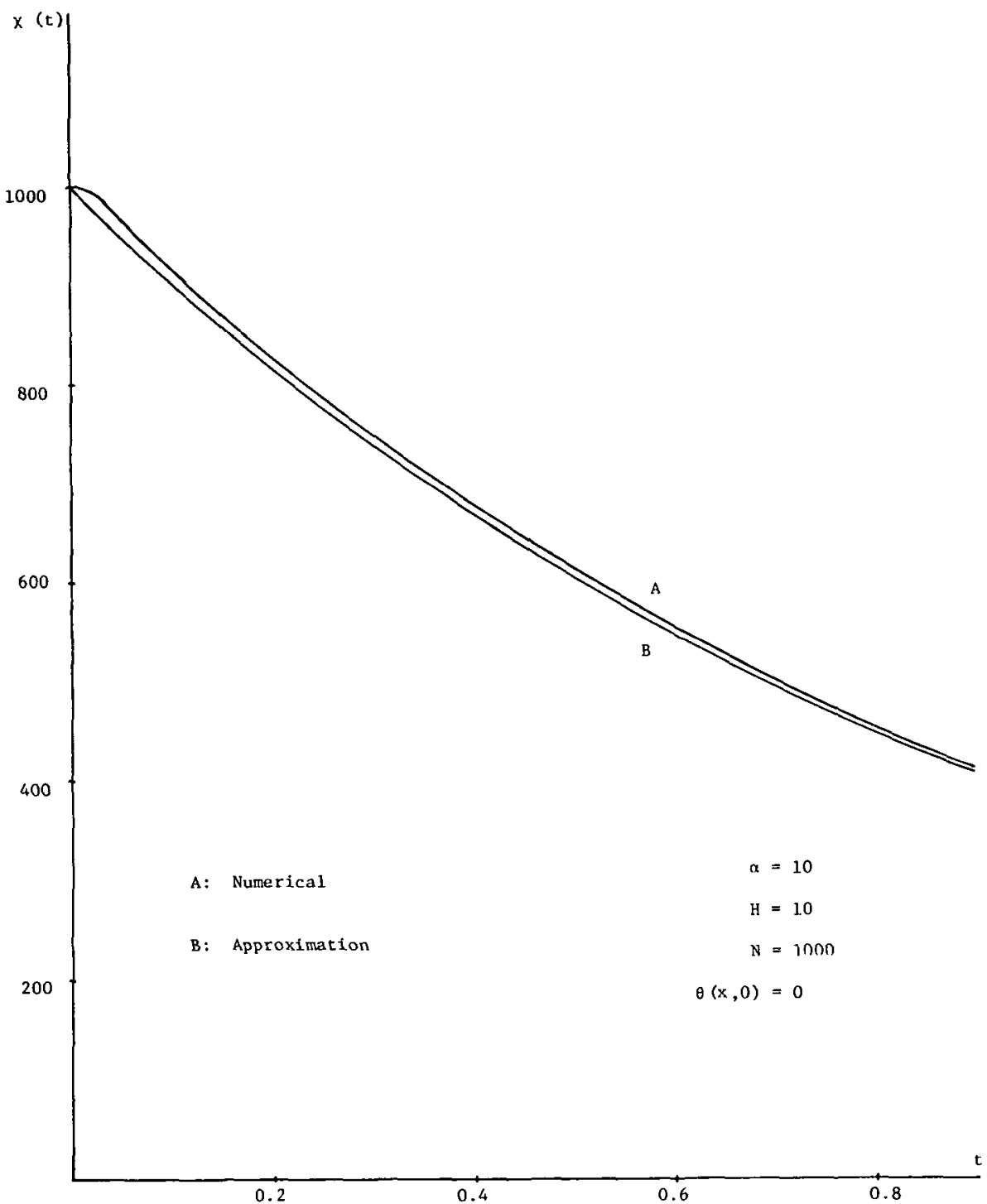

Figure 1. The quantity $\chi(t)$ as a function of $t$ for the infinite slab.

Combining the two expressions, we have

$$
\begin{aligned}
& \varepsilon \theta+H \chi \\
& \doteqdot H N\left\{\frac{\cos \left(x-\frac{1}{2}\right)}{\cos \frac{1}{2}} e^{-1}-\sum_{n=0}^{\infty} \frac{(-1)^{n} 4 \cos \left[(2 n+1) \pi\left(x-\frac{1}{2}\right)\right]}{(2 n+1) \pi\left[(2 n+1)^{2} \pi^{2}-1\right]} e^{-(2 n+1)^{2} \pi^{2} t}\right\},
\end{aligned}
$$




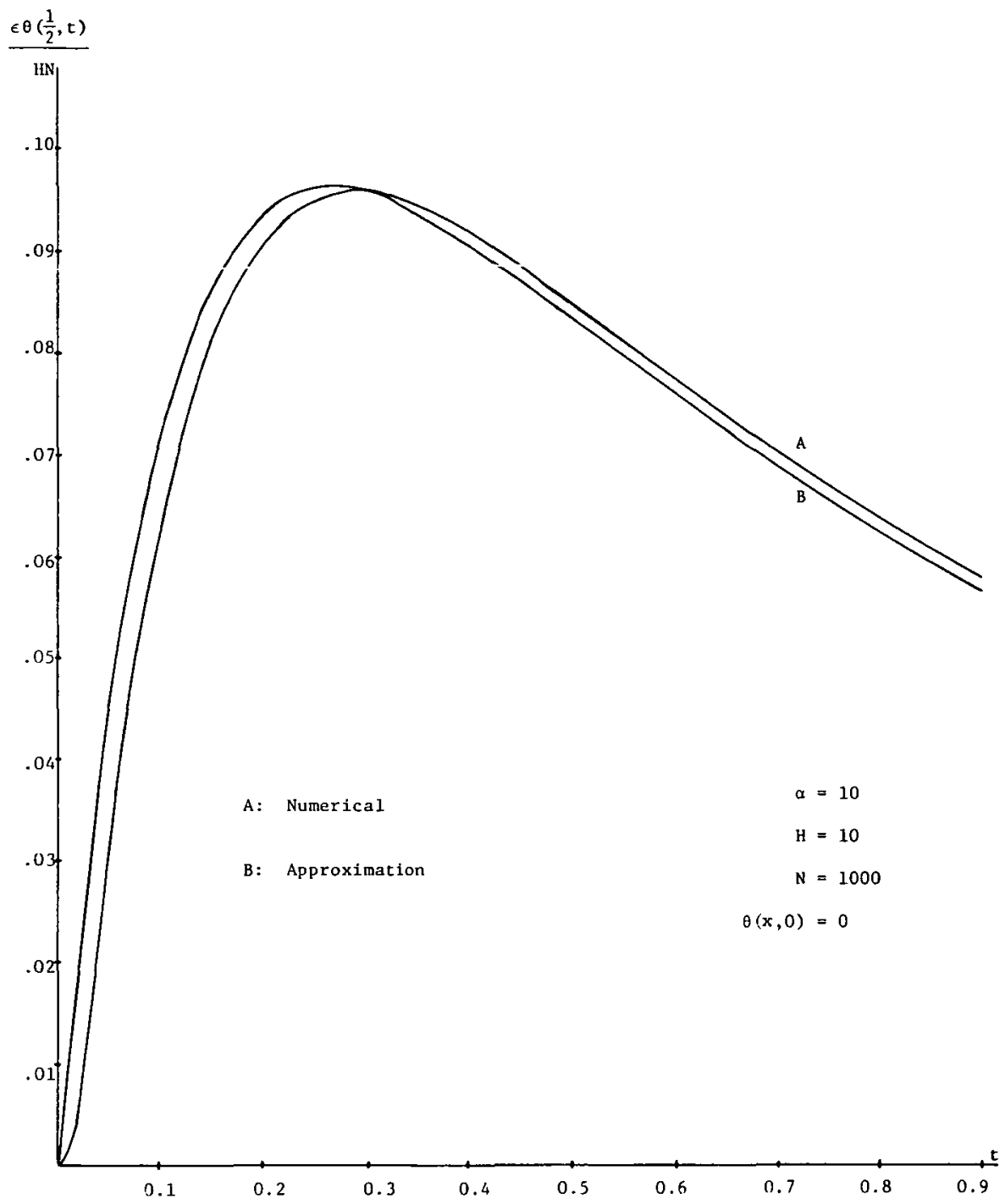

Figure 2. The quantity $\varepsilon \theta\left(\frac{1}{2}, t\right) / H N$ as a function of $t$ for the infinite slab.

\section{from which follows}

$$
\varepsilon \theta\left(\frac{1}{2}, t\right) \doteqdot H N\left\{0.139494 e^{-t}-\sum_{n=0}^{\infty} \frac{(-1)^{n} 4 e^{-(2 n+1)^{2} \pi^{2} t}}{(2 n+1) \pi\left[(2 n+1)^{2} \pi^{2}-1\right]}\right\}
$$


The time $t_{m}$ at which $\theta\left(\frac{1}{2}, t\right)$ acquires its maximum can be readily obtained. We observe that $t_{m}$ is independent of $\varepsilon, H$ and $N$ to this order of approximation. Using the expression for $\theta\left(\frac{1}{2}, t\right)$ in (11), we can calculate an improved description for $\chi(t)$ from (9). The integral has to be evaluated numerically, but the multiple time scale feature is apparent. While the iteration can be carried forward in principle, no such attempt will be made.

To see how well (11) approximates the true solution, we solve equations (1) and (2) numerically, for $0<x<1, h(x)=0, g(x)=N=1000, \alpha=10, H=10$. It is found that $\chi$ is practically independent of the spatial variable so that the assumption of equation (10) is justified. In Figures 1 and 2 the comparisons for $\chi$ and $\varepsilon \theta\left(\frac{1}{2}, t\right) / H N$ are presented for $0<t<0.9$.

\section{Approximations for $\theta$ and $\chi$ for the sphere}

Assuming spherical symmetry, with $0<r<1$, and using the same notation as in Section 2, we have

$$
\begin{aligned}
\frac{d^{2} \bar{\varphi}}{d r^{2}}+\frac{2}{r} \frac{d \bar{\varphi}}{d r}-p \bar{\varphi} & =-H N, \\
\bar{\varphi}(1, p) & =H \bar{\chi}_{s},
\end{aligned}
$$

from which we obtain

$$
\bar{\varphi}(r, p)=\frac{H N}{p}+\left(H \bar{\chi}_{s}-\frac{H N}{p}\right) \frac{\sin i \sqrt{p} r}{r \sin i \sqrt{p}} .
$$

To invert the above, we introduce the same simplification on $\chi$ as in Section 2 . Then we have

$$
\varphi(r, t) \doteqdot H N\left\{\frac{\sin r}{\sin 1} e^{-t}+2 \sum_{n=1}^{\infty} \frac{(-1)^{n}}{n \pi\left(n^{2} \pi^{2}-1\right)} \frac{\sin n \pi r}{r} e^{-n^{2} \pi^{2} t}\right\}
$$

and in particular

$$
\varepsilon \theta(0, t) \doteqdot H N\left\{0.188395 e^{-t}+2 \sum_{n=1}^{\infty} \frac{(-1)^{n}}{n^{2} \pi^{2}-1} e^{-n^{2} \pi^{2} t}\right\} .
$$

An improved approximation for $\chi$ is as given in (9).

To provide a comparison, we solve the governing equations numerically for the sphere with $0<r<1, h(r)=0, g(r)=N=1000, \alpha=10, H=10$. In Figure 3, the quantity $\varepsilon \theta(0, t) / H N$ given by (12) is compared with the corresponding numerical result. 


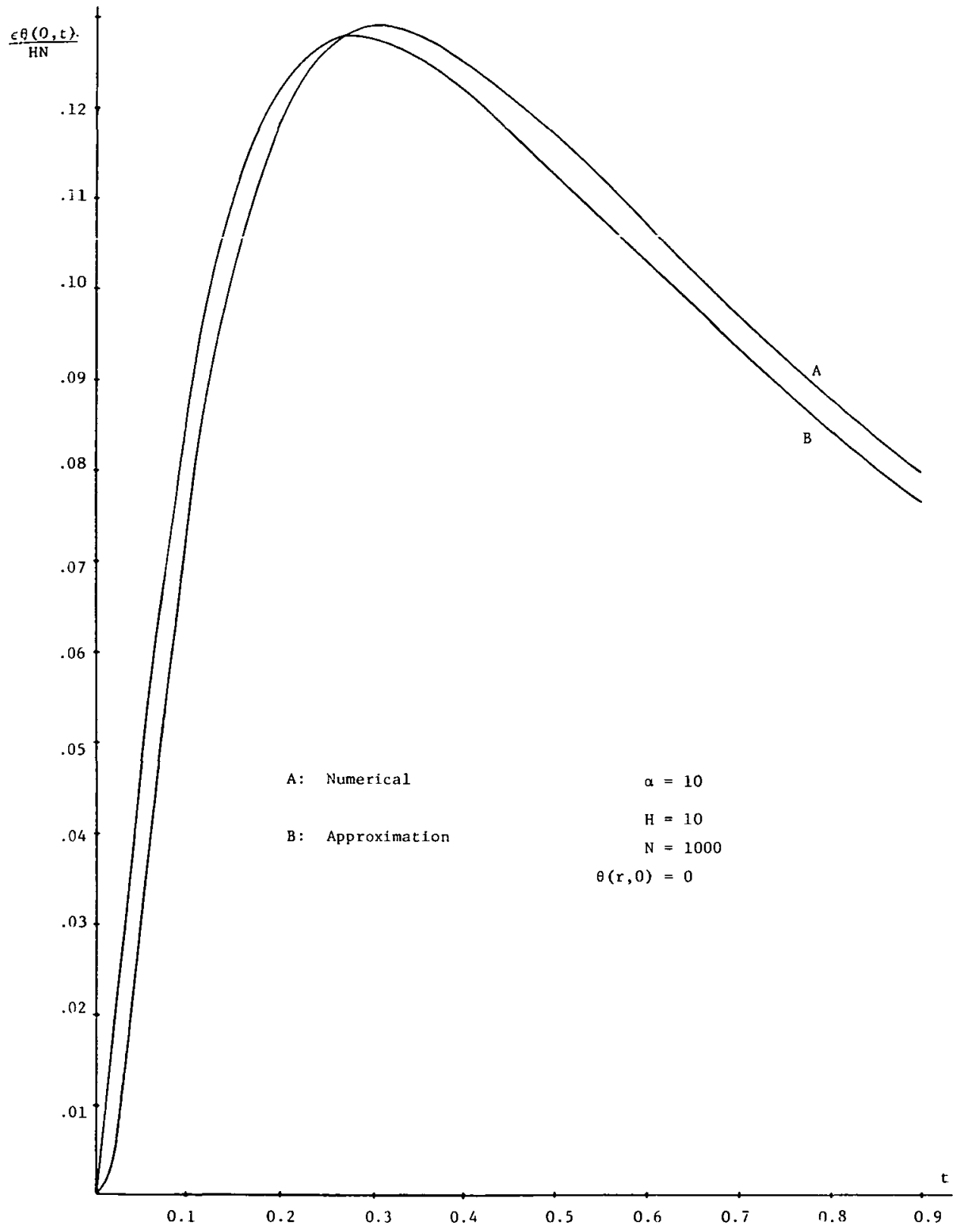

Figure 3. The quantity $\varepsilon \theta(0, t) / H N$ as a function of $t$ for the sphere. 


\section{Approximations for $\theta$ and $\chi$ for the infinite cylinder}

Assuming cylindrical symmetry, with $0<r<1$, and with the same notation as before, we have

$$
\begin{aligned}
\frac{d^{2} \bar{\varphi}}{d r^{2}}+\frac{1}{r} \frac{d \bar{\varphi}}{d r}-p \bar{\varphi} & =-H N \\
\bar{\varphi}(1, p) & =H \bar{\chi}_{s},
\end{aligned}
$$

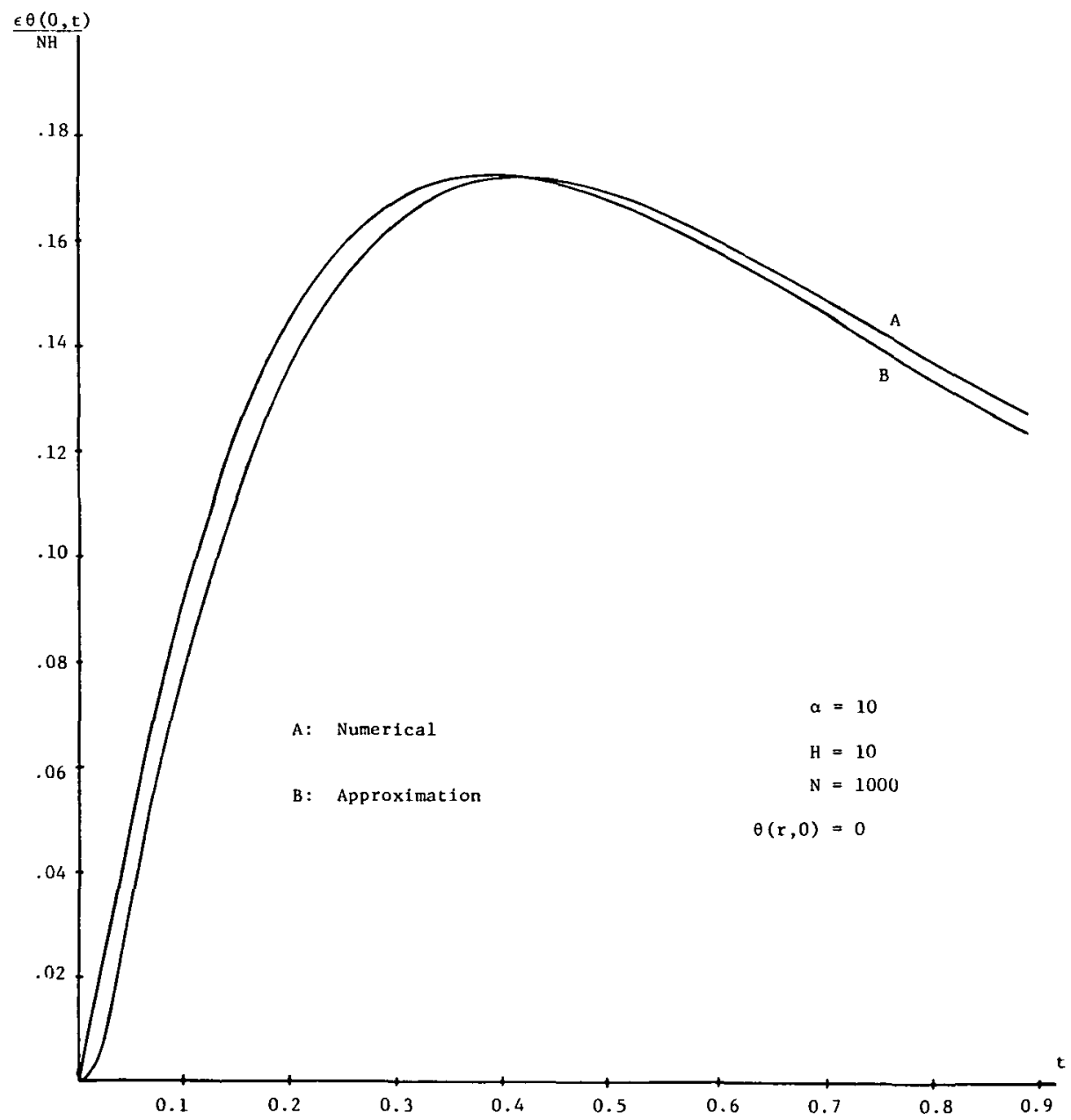

Figure 4. The quantity $\varepsilon \theta(0, t) / H N$ as a function of $t$ for the infinite cylinder. 
from which we obtain

$$
\bar{\varphi}(r, p)=\frac{H N}{p}+\left(H \bar{\chi}_{s}-\frac{H N}{p}\right) \frac{J_{0}(i \sqrt{p} r)}{J_{0}(i \sqrt{p})} .
$$

Using the same simplification on $\chi$, we obtain

$$
\varphi(r, t) \doteqdot H N\left\{\frac{J_{0}(r)}{J_{0}(1)} e^{-t}-2 \sum_{n=1}^{\infty} \frac{1}{k_{n}\left(k_{n}^{2}-1\right)} \frac{J_{0}\left(k_{n} r\right)}{J_{1}\left(k_{n}\right)} e^{-k_{n}^{2} t}\right\},
$$

where $k_{n}, n=1, \ldots$, are the zeros of $J_{0}(r)$. In particular, we have

$$
\varepsilon \theta(0, t) \doteqdot H N\left\{0.30685 e^{-t}-2 \sum_{n=1}^{\infty} \frac{1}{k_{n}\left(k_{n}^{2}-1\right)} \frac{e^{-k_{n}^{2} t}}{J_{1}\left(k_{n}\right)}\right\} .
$$

An improved description for $\chi$ is as given in (9).

Again, we solve the governing equations numerically for the infinite cylinder with $0<r<1, h(r)=0, g(r)=N=1000, \alpha=10, H=10$. In Figure 4, we compare $\varepsilon \theta(0, t) / H N$ obtained from (13) and from the numerical solution.

\section{Concluding remarks}

For each geometry, we have constructed an approximation for $\theta$ whose dependence on $\varepsilon, H$ and $N$ is explicitly demonstrated. As seen from the plots presented, the approximation agrees well with the numerical result. The relative ease with which the result is obtained depends crucially on the initial approximation for $\chi$, which in turn is based on the assumption of rapid transient phenomenon in $\theta$. The nature of the approximation suggests that the accuracy of the expressions for $\theta$ and $\chi$ will decrease as time increases. This is not serious, however, as the main interest here is the description of the rapid transient behaviour.

We have dealt with the case of $\theta(x, 0)=0$, but the influence of a non-zero $\theta(x, 0)$ can be studied without much difficulty. In particular, if $\theta(x, 0)=K=$ constant, our results holds if $H N$ is replaced by $H N+\varepsilon K$ in the expression for $\varphi$.

The case of higher order reaction can be treated in a similar manner, and is under study. It is clear that $\chi$ will decay algebraically instead of exponentially and the corresponding expression for $\theta$ will be more difficult to obtain. 


\section{Acknowledgements}

This research was supported by the Natural Science and Engineering Research Council of Canada under Grant S-5228. The author also thanks Mr. D. Collins for obtaining the numerical solutions.

\section{References}

[1] D. A. Frank-Kamenetskii, Diffusion and heat transfer in chemical kinetics (Translation editor J. P. Appleton), (Plenum Press, New York, 1959).

[2] I. M. Gelfand, "Some problems in the theory of quasi-linear equations", AMS Translations Ser. 2, 29(1963), 295-381.

[3] D. R. Kassoy, "Extremely rapid transient phenomena in combustion, ignition and explosion", SIAM-AMS Proceedings 10 (1976), 61-72.

[4] J. R. Parks, "Criticality criteria for various configurations of a self-heating chemical as functions of activation energy and temperature of assembly", J. Chem. Phys. 34 (196I), $46-50$.

[5] D. H. Sattinger, "A nonlinear parabolic system in the theory of combustion", Quart. Appl. Math. 33 (1975), 47-61.

[6] K. K. Tam, "Construction of upper and lower solutions for a problem in combustion theory", J. Math. Anal. Appl. 69 (1979), 131-145.

[7] K. K. Tam, "Initial data and criticality for a problem in combustion theory",J. Math. Anal. Applic. 77 (1980), 626-634.

[8] K. K. Tam, "On the influence of the initial data in a combustion problem", J. Austral. Math. Soc. Ser. B 22 (1980), 193-209.

[9] K. K. Tam, "Computation of critical parameters for a problem in combustion theory ", $J$. Austral. Math. Soc. Ser. B 24 (1982), 40-46. 